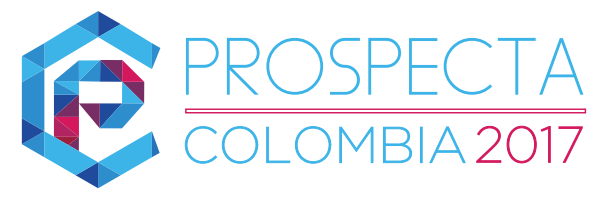

\title{
Modelo de gestión de proyectos para una pyme del sector de servicios de sonido e iluminación profesional en Colombia
}

Model management of projects for a SME of the sound and lighting services sector in Colombia

Juan Gabriel Cabrera Ortiz*

* juang.cabrera@unad.edu.co 


\section{Introducción}

En el país, actualmente las pymes del sector de servicios enfrentan el reto de desarrollar e implementar proyectos encaminados al cumplimiento del plan estratégico y de los objetivos organizacionales (Guerrero, 2013). Además, un estudio denominado "Las pyme: Una mirada a partir de la experiencia académica del MBA PYME" plantea que estas deben alinearse con las tendencias que impone la gerencia del siglo XXI, en donde la competitividad se convierte en el eje central del negocio, la producción del conocimiento en el factor determinante para transformar este tipo de empresas y estimular, entre otros: la creatividad, la generación de conocimientos y la creación de productos y servicios (Villegas \& Iván, 2010). Es bien sabido que la gestión de proyectos es una pieza fundamental para la consecución de los objetivos y el alcance de los proyectos, por lo que se consolida como una actividad importante para las organizaciones dedicadas a la ingeniería (Buchtik, 2012). Se entiende por gestión de proyectos al método que permite la definición, priorización y ejecución de proyectos dentro de un marco de prácticas; expresa los valores o sistemas de valores que mantienen la organización y sus miembros; se basa en la aplicación de modelos de diseño y ejecución; además se constituye en un entorno en el que, mediante el trabajo en grupos, los individuos cumplen eficiente y eficazmente los objetivos de los proyectos (Lazaro, Laverde y Guacheta, 2013). Esto hace que cada vez se otorga una mayor importancia al desempeño de esta área del conocimiento, dado que permite lograr una optimización con grandes beneficios para la organización (Rodríguez, Aguilar \& Sánchez, 2012). Teniendo en cuenta lo anterior, se plantea el desarrollo de un modelo para la gestión de proyectos de una pyme del sector de servicios de alquiler de sistemas de sonido e iluminación profesional.

La pyme seleccionada es Ruffo Sánchez Amplificación Profesional Ltda. (RSAP). Esta empresa realiza entre 220 y 240 proyectos al año, sin embargo en la actualidad no se encuentra certificada en ningún sistema de gestión de la calidad (Sánchez, 2015) ni hace uso del enfoque de las buenas prácticas del PMI (Instituto para la Gestión de Proyectos, por sus siglas en inglés). Por ende, es importante construir un perfil que permita desarrollar sus capacidades y 
entender sus necesidades, teniendo en cuenta los enfoques de gestión y madurez en proyectos. Esta última se debe entender como la capacidad que tiene una organización, proceso o área para reconocer su punto de desarrollo en comparación con un estándar (Solarte y Sánchez, 2014).

\section{Marco teórico}

Pyme: Ruffo Sánchez Amplificación

Profesional Ltda.

Ruffo Sánchez Amplificación Profesional es una empresa de sociedad limitada constituida legalmente desde el año 1992. La empresa, ubicada en la ciudad de Bogotá, se dedica a la producción y realización de eventos, activaciones de marca, BTL, sonido, luces, etc. Su misión es "brindar soluciones audaces, en la producción y realización de eventos de éxito" y su visión posicionarse "como una compañía ágil, versátil y eficaz en la producción, planeación, organización y realización de eventos en Colombia". RSAPL no cuenta con ninguna certificación de calidad o en ningún otro tipo de sistema de gestión (Sánchez R, 2015); no obstante, tiene aproximadamente 37 empleados y realiza entre 220 y 240 eventos en el año. Algunos de sus actuales clientes son General Motors Company, RCN Televisión, Pidamos Eventos y Promociones, Caracol Televisión, Chevrolet, Telecolombia S.A., Presidencia de la Republica, Comité Olímpico Colombiano, Almacenes Éxito, Sístole, Hyundai, Bayer, Corferias, Totto, entre otros.

\section{Proyecto}

Un proyecto es un esfuerzo temporal de desempeño gradual emprendido para crear un entregable. Es temporal porque tiene un comienzo y un fin definidos; el inicio se da cuando es aprobado y el fin cuando termina, ya sea que se logren o no los objetivos planteados. Se crea un entregable que es único en su género, diferente en forma perceptible de los demás entregables y que no se ha realizado anteriormente de la misma forma (PMI, 2008; Crowe, 2011; Johnson, 2007 y Mulcahy, 2009). 
Ciclo de vida del proyecto

"Es la serie de fases por las que atraviesa un proyecto desde su inicio hasta su cierre. Las fases son generalmente secuenciales y sus nombres y números se determinan en función de las necesidades de gestión y control de la organización u organizaciones que participan en el proyecto, la naturaleza propia del proyecto y su área de aplicación" (PMBOK, 2013, p. 38). Las fases se pueden dividir por objetivos funcionales o parciales, resultados o entregables intermedios, hitos específicos dentro del alcance global del trabajo o disponibilidad financiera. Las fases son generalmente acotadas en el tiempo, con un inicio y un final o punto de control. Un ciclo de vida se puede documentar dentro de una metodología. Se puede determinar o conformar el ciclo de vida del proyecto sobre la base de los aspectos únicos de la organización, de la industria o de la tecnología empleada. Mientras que cada proyecto tiene un inicio y un final definidos, los entregables específicos y las actividades que se llevan a cabo variarán ampliamente dependiendo del proyecto. El ciclo de vida proporciona el marco de referencia básico para dirigir el proyecto, independientemente del trabajo específico involucrado (PMBOK, 2013).

\section{Gestión de proyectos}

Para el PMI (2013) la dirección de proyectos es la aplicación de conocimientos, habilidades, herramientas y técnicas a las actividades del proyecto para cumplir con los requisitos del mismo. Se logra mediante la aplicación e integración adecuadas de los 47 procesos de la dirección de proyectos, agrupados de manera lógica, categorizados en los siguientes cinco grupos de procesos (PMBOK, 2013):

- Inicio

- Planificación

- Ejecución

- Monitoreo y control

- Cierre 
Adicionalmente, Nokes (2006) define la dirección de proyectos como la disciplina del planeamiento, la organización, la motivación y el control de los recursos con el propósito de alcanzar uno o varios objetivos con un principio y un final definidos (normalmente limitado en tiempo, en costos o entregables), que es emprendido para alcanzar objetivos únicos y que dará lugar a un cambio positivo o agregará valor (Nokes, 2006).

Guía de fundamentos para la gestión de proyectos Guía del PMBOK® (quinta edición)

EI PMBOK® define a la gestión de proyectos como "la aplicación de conocimientos, habilidades, herramientas y técnicas a las actividades del proyecto para cumplir con los requisitos del mismo" (PMBOK, 2013). Esto se logra a través de la aplicación de 47 procesos de dirección de proyectos, referenciados en un orden lógico y categorizados en los 5 grupos de procesos (inicio, planificación, ejecución, monitoreo y control, cierre), (PMBOK, 2013).

Los 47 procesos de la dirección de proyectos se agrupan a su vez en diez (10) áreas del conocimiento. Un área del conocimiento es la representación de un conjunto de conceptos términos y actividades que conforman un ámbito en la dirección de proyectos o una línea de especialización. Estas áreas del conocimiento se utilizan en la mayoría de los proyectos. (PMBOK, 2013).

\section{Modelos de madurez en la gestión de proyectos}

En la actualidad, existen varios estándares de madurez en materia de la gestión de proyectos, estos han sido considerados como el mecanismo con que una organización debe contar para reflejar que está involucrada en la práctica de los mejores procesos, en procura de la excelencia en la administración de proyectos (López, 2013). Se presenta el modelo de Haorld Kerzner, modelo escogido por sus ventajas en la aplicabilidad en PYMES. 
Modelo de madurez en gestión de proyectos de Harold Kerzner - PMMM

El modelo de Kerzner propone 5 niveles de madurez (lenguaje común, procesos comunes, metodología singular, benchmarking, mejoramiento continuo) en función de las mejores prácticas en la gestión de proyectos. Kerzner afirma que los niveles de madurez de una organización pueden traslaparse entre sí, por ejemplo, los niveles 1 y 2 pueden desarrollarse paralelamente; sin embargo, para alcanzar el tercer nivel se debe alcanzar la madurez requerida en dichos niveles. Para el diagnóstico de cada nivel de madurez existe un instrumento específico que mide las buenas prácticas en gestión de proyectos. Adicionalmente, en el Modelo de Kerzner se establecen tres niveles de riesgo (bajo, medio y alto) para cada nivel del modelo. En este sentido, cada nivel conlleva cierto nivel de riesgo para ser desarrollado, y el riesgo tiene que ver con el impacto al cambio de la cultura corporativa en la organización. (Kerzner, 2002).

\section{Modelos de organización}

Este concepto se refiere a una representación en la organización que evidencie los componentes de un sistema o subsistema de la misma (unidades, sectores, funciones y servicios) y la relación entre ellos a través de procesos y tareas repetibles (Rivas, 2002). El análisis de "lo actual" le permite a la dirección de la organización evaluar la correspondencia con lo deseado, encontrar las brechas de estructura que impiden una correcta operación, como la desconexión del contexto, la duplicación de funciones y otros aspectos disfuncionales de la organización (Mintzberg, 1984). En este sentido, el modelo en una organización debe ser un instrumento útil para el análisis y la gestión de la organización, y este debe ser capaz de servir como guía y proyección para definir las formas ideales de la de articulación de unidades, funciones y grupos en la organización (Etkin, 2009). 


\section{Subjetividad de los modelos}

Al analizar o construir modelos al interior de una organización se debe considerar que se está tratando con un sistema social, por lo tanto, en él actuarán factores subjetivos como los fines, motivaciones, expectativas e interpretaciones que se incorporan al diálogo y a la interacción social. Aunque puede existir una versión oficial del modelo provisto desde la dirección, existe también una visión propia y particular de los integrantes de la organización (Mintzberg, 1984). La subjetividad hace la diferencia entre estructura y modelo, estructura supone las formas manifiestas de ordenar un grupo de actividades en términos de autoridad y reglas de operación, un modelo supone una estructura mental, una forma de pensar y de hacer bajo cierto contexto, el modelo se refiere a pautas de relación asumidas que se aprenden y se construyen en la interacción social (Etkin, 2009).

\section{Valor del modelo: representación y pertinencia}

Thierauf (1985) define al modelo como "una representación o abstracción de una situación u objetos reales, que muestra las relaciones y las interacciones en términos de causa, efecto y reciprocidad" y "para que este sea completo como método, el modelo debe ser representativo de aquella realidad que se está analizando y se quiere formalizar" (Thierauf, 1985), es decir, en el modelo se deben encontrar las variable críticas o pertinentes del sistema de gestión. Como principio y criterio básico se puede hablar sobre la congruencia de un modelo con cierta situación, pero no es posible definir con un carácter general el mejor modelo. En este sentido, el alcance de un modelo debe entenderse en su contexto, su espacio y su tiempo, desde esa posición se puede calificar a un modelo como congruente, pertinente y adecuado y también si es aceptable o no en término económicos, políticos o sociales. Lo anterior requiere probar el diseño a razón de las prioridades internas pero también correlacionarlo con las demandas y las condiciones de su contexto (Rivas, 2002). 


\section{Metodología}

La investigación se enmarca en el tipo de investigación descriptiva dado que el problema es identificado con claridad; sin embargo se requiere información adicional para poder llegar a establecer caminos que conduzcan al esclarecimiento de las relaciones causales y su posible solución (Jiménez, 1998). Se emplea el método empírico dado que los resultados son producto de las experiencias obtenidas y estos permiten identificar las relaciones y características fundamentales del objeto de estudio (Jiménez, 1998).

Además de lo anterior, los enfoques de investigación cualitativo y cuantitativo se complementan en el conocimiento, explicación y comprensión de las realidades, estos ofrecen elementos importantes, tienen límites y distintas posibilidades (Monje, 2011). La combinación de los dos enfoques de investigación, y su articulación en las distintas etapas, permiten y sustentan el desarrollo del modelo de gestión de proyectos propuesto y la validación del mismo. A continuación, se relacionan las fuentes de información y la ruta metodología a emplear para el desarrollo de cada uno de las etapas del trabajo (tabla 1).

\section{Tabla 1. Metodología}

\begin{tabular}{ccc}
\hline Etapa del Proyecto & Metodología & Productos a Obtener \\
\hline $\begin{array}{c}\text { Diagnóstico del } \\
\text { perfil de madurez de } \\
\text { la pyme objeto de } \\
\text { estudio }\end{array}$ & $\begin{array}{c}\text { Aplicación de instrumentos a la } \\
\text { Kuestra definida según el modelo de } \\
\text { información. Análisis y presentación de } \\
\text { resultados. }\end{array}$ & $\begin{array}{c}\text { Perfil de madurez en } \\
\text { relación al modelo de } \\
\text { madurez seleccionado }\end{array}$ \\
\hline $\begin{array}{c}\text { Desarrollo del modelo } \\
\text { para la gestión de } \\
\text { proyectos en la pyme }\end{array}$ & $\begin{array}{c}\text { Consulta a expertos, revisión de } \\
\text { documentos, análisis de la información, } \\
\text { elaboración y ajuste del modelo }\end{array}$ & $\begin{array}{c}\text { Modelo para la gestión de } \\
\text { proyectos para la pyme }\end{array}$ \\
\hline $\begin{array}{c}\text { Validación por } \\
\text { expertos del modelo } \\
\text { desarrollado }\end{array}$ & $\begin{array}{c}\text { Consulta a expertos, realimentación, } \\
\text { ajustes, validación de ajustes }\end{array}$ & $\begin{array}{c}\text { Modelo para la gestión } \\
\text { de proyectos validado por } \\
\text { expertos }\end{array}$ \\
\hline
\end{tabular}

Fuente: elaboración propia 
Para el diagnóstico del perfil de madurez se aplica el modelo de Harold Kerzner en la metodología, instrumentos de evaluación y análisis de resultados. Luego, haciendo uso de técnicas de recolección de información cuantitativas y cualitativas, se procede a establecer la caracterización de los proyectos de RSAP y el flujo de actividades, procesos y procedimientos realizados en la gestión de sus proyectos, junto con las herramientas y tecnología implementadas. Posteriormente, y con base en los conocimientos que se referencian en la guía de fundamentos para la dirección de proyectos del PMI, se plantea un modelo que tenga en cuenta el perfil de madurez actual y los demás elementos enunciados. Por último, se valida el modelo desarrollado por medio del criterio de expertos en función de cuatro categorías, suficiencia, claridad, coherencia y relevancia.

\section{Desarrollo}

Diagnóstico de madurez según el modelo de Harold Kerzner P3M

Harold Kerzner define al modelo P3M (PMMM) como la base para lograr la excelencia en la gestión de proyectos, en donde cada nivel definido en el modelo representa un grado ascendente en el alcance de la excelencia. Aunque en el modelo $\mathrm{P}_{3} \mathrm{M}$ puede existir solapamiento entre algunos niveles, el orden en el que se proponen los niveles no se debe cambiar. En este sentido, la superposición se puede dar entre los niveles 1-2, 3-4 y 4-5; por ende, para lograr el tercer nivel necesariamente se deberán alcanzar los niveles 1 y 2 . En la figura 1 se evidencia el solapamiento de niveles que se propone en el modelo (Kerzner, 2002). 


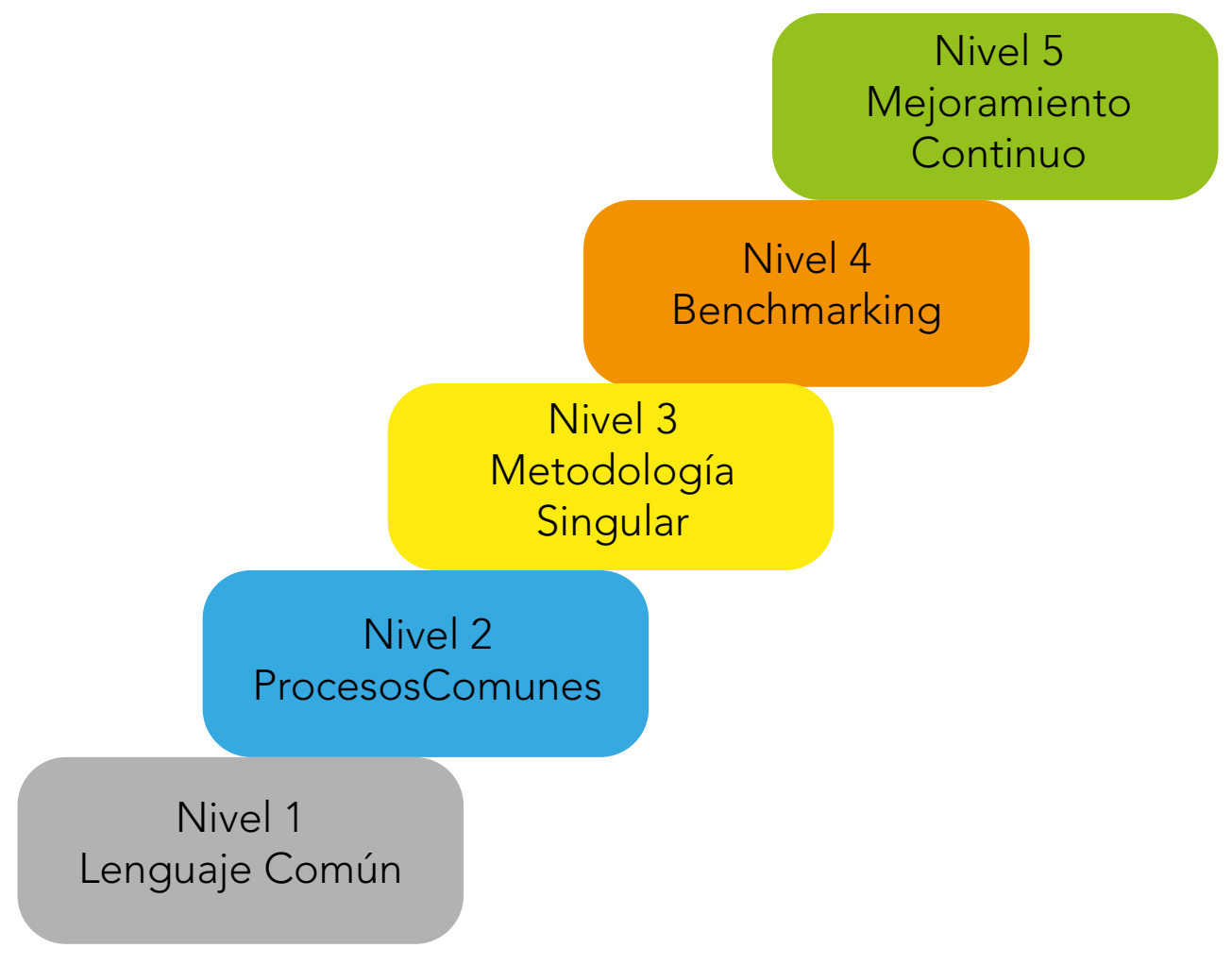

Figura 1. Solapamiento entre niveles de Madurez Kerzner Fuente: Kerzner, (2002)

\section{Selección de muestra en la organización objeto de estudio y diagnóstico de} madurez

Se realiza una revisión de la estructura organizacional actual de la pyme, con el fin de definir dos personas de diferentes niveles de autoridad (nivel alto, nivel medio). Esto con el fin de realizar la aplicación de los instrumentos de evaluación de madurez que el modelo establece. En la figura 2 se evidencia la estructura organizacional de la pyme. 


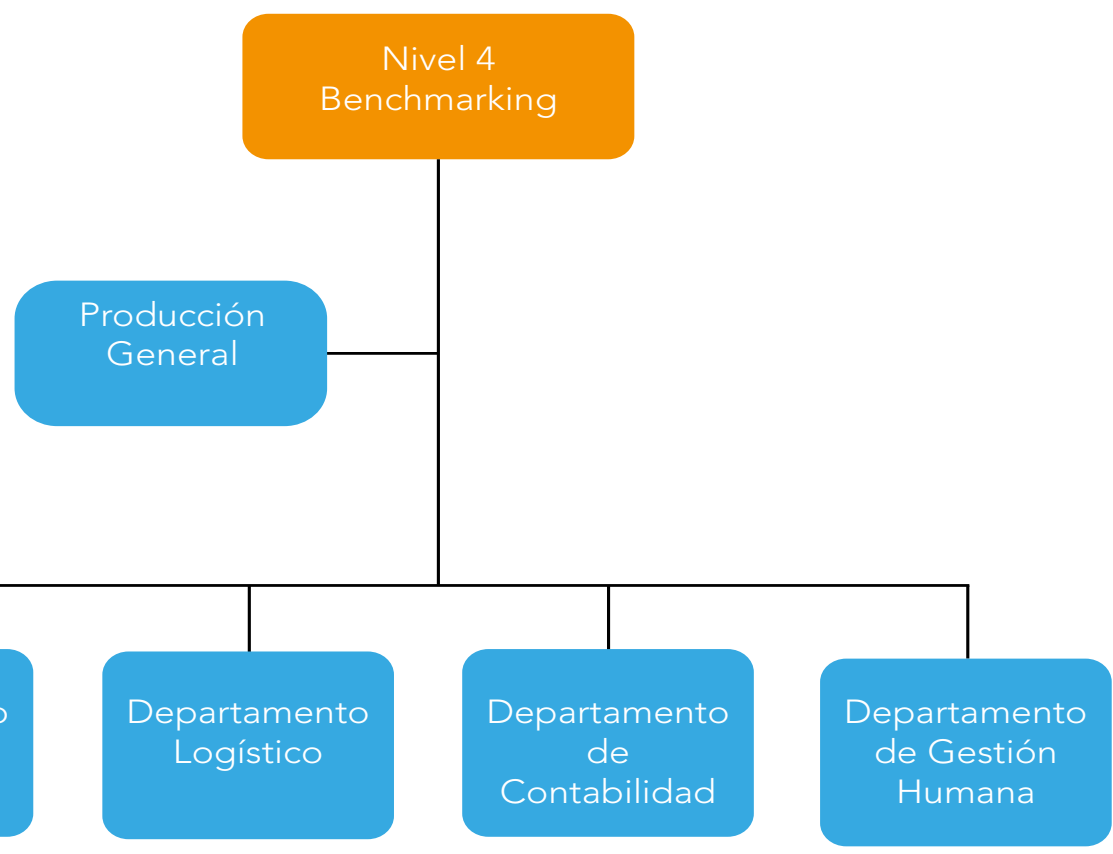

Figura 2. Estructura organizacional RSAP

Fuente: elaboración propia

Los funcionarios de RSAP a los cuales se les aplica el instrumento pertenecen a los niveles jerárquicos 2 y 3 , garantizando que estos cuentan con amplia trayectoria en la compañía y conocen bien los procesos funcionales de la misma.

Evaluación de madurez nivel 1. Lenguaje común

Para el diagnóstico de madurez del nivel 1 se aplica el instrumento correspondiente a los funcionarios seleccionados. Para aprobar el nivel 1, el modelo establece obtener un total de Go puntos o más por área. A continuación, se relacionan los resultados obtenidos en la evaluación del nivel 1 (tabla 2, figura 3). 
Tabla 2. Resultados por área evaluada en el nivel de madurez 1 - Lenguaje común

\begin{tabular}{ll}
\hline Área evaluada & Puntaje final \\
\hline Gestión del alcance & 55 \\
\hline Gestión del tiempo & 40 \\
\hline Gestión de costo & 35 \\
\hline Gestión de recursos humanos & 40 \\
\hline Gestión de las adquisiciones & 25 \\
\hline Gestión de la calidad & 25 \\
\hline Gestión del riesgo & 30 \\
\hline Gestión de las comunicaciones & 45 \\
\hline Total & 295
\end{tabular}

Fuente: elaboración propia

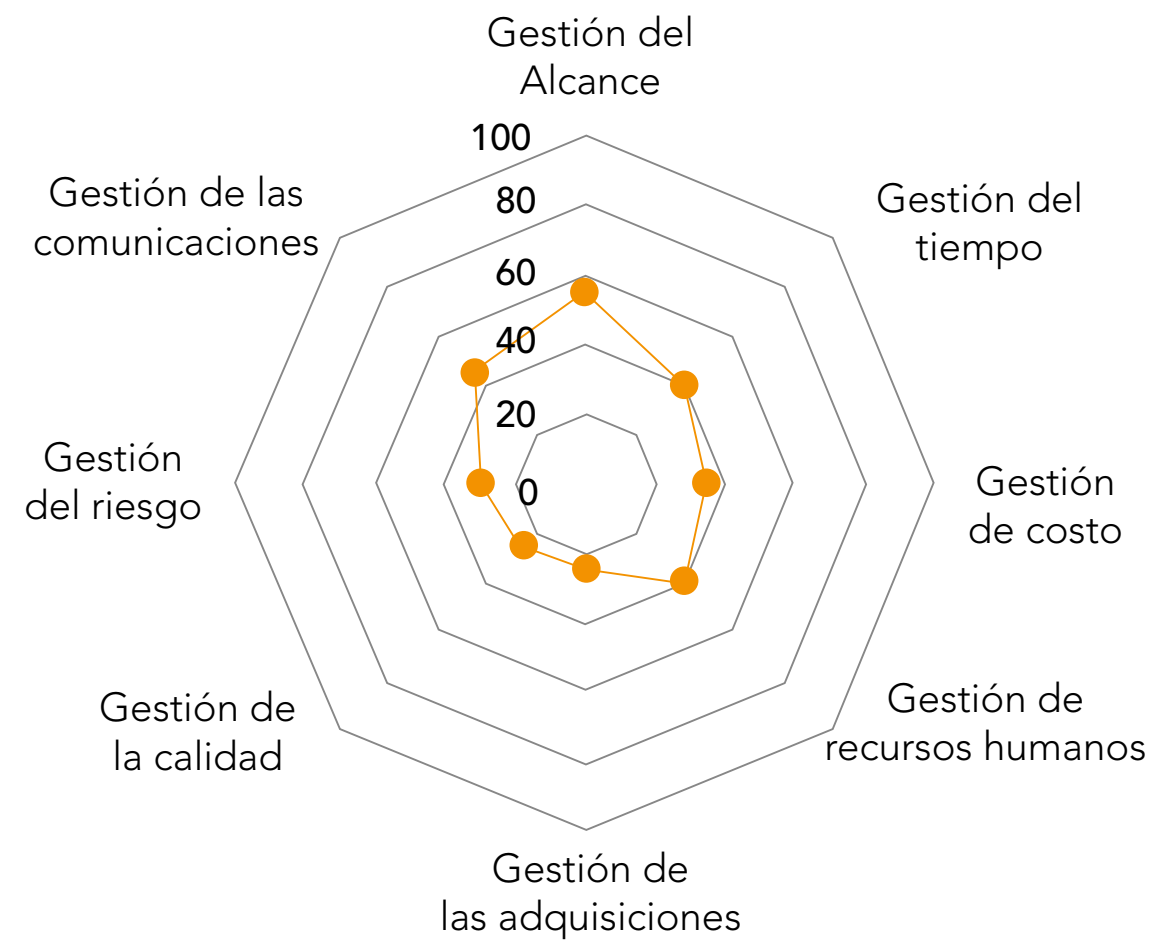

Figura 3. Perfil de madurez RSAP nivel 1 - Leguaje común

Fuente: elaboración propia 
En términos de puntaje general, la organización logró obtener 295 puntos. Con base en la suma del puntaje final obtenido en cada área, cabe resaltar que el nivel 1 no está superado, según lo expuesto por Kerzner (2002), pues para hacerlo se requieren 600 puntos o más.

Evaluación de madurez nivel 2 - Procesos comunes Según Kerzner (2002), este nivel se plantea desde un enfoque de cinco fases del ciclo de vida como se evidencia en la figura 4. Para que se considere que la organización estudiada supere o se ubique en una de estas fases de este nivel de madurez, se requiere obtener un puntaje mínimo de 6 para cada fase del ciclo de vida.

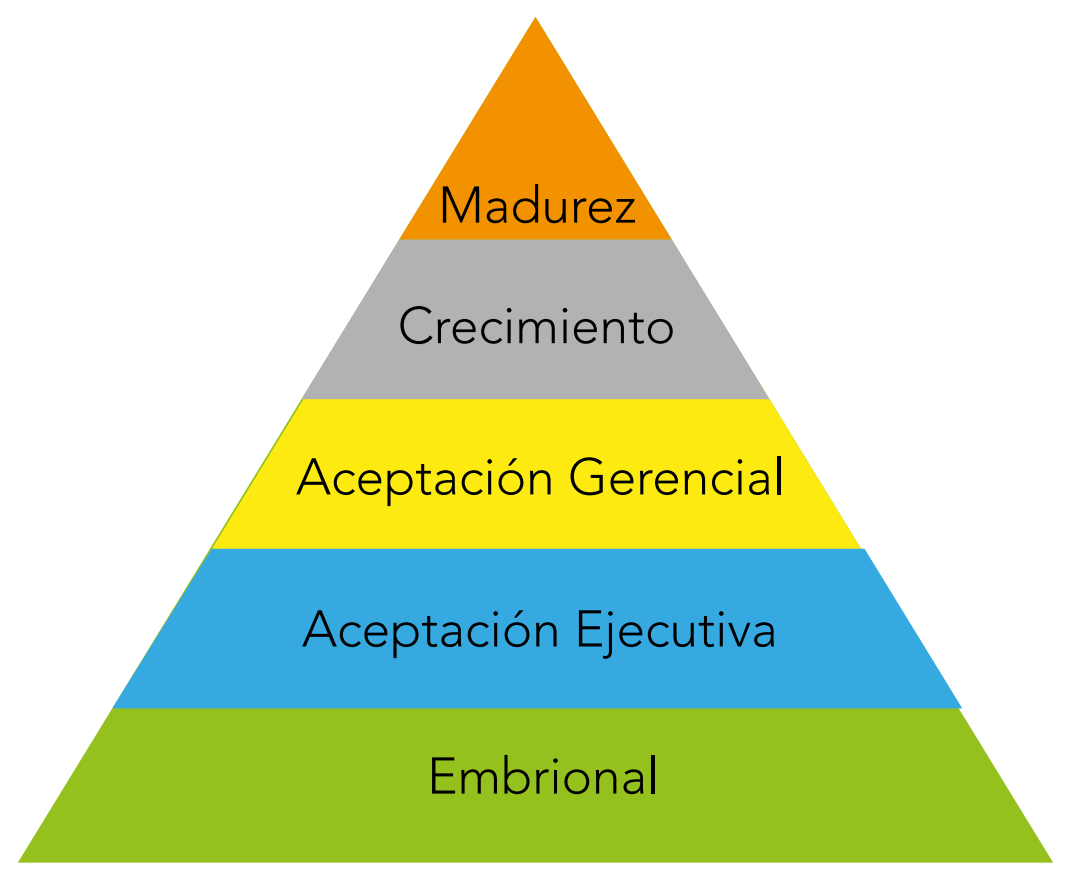

Figura 4. Fases del Ciclo de Vida Nivel 2 - Kerzner.

Fuente: Kerzner, (2002). 
En la tabla 3 se presentan los resultados obtenidos para el diagnóstico de este nivel de madurez.

Tabla 3. Puntaje obtenido en el nivel 2 - Proceso comunes

\begin{tabular}{|c|c|c|c|c|c|c|c|c|c|c|c|c|}
\hline & $-12-10$ & -8 & -6 & -4 & -2 & 0 & 2 & 4 & 6 & 8 & 10 & 12 \\
\hline Madurez & & $x$ & & & & & & & & & & \\
\hline Crecimiento & & & $x$ & & & & & & & & & \\
\hline Aceptación gerencial & & & & $x$ & & & & & & & & \\
\hline Aceptación ejecutiva & & & & & $x$ & & & & & & & \\
\hline Embrional & & $x$ & & & & & & & & & & \\
\hline
\end{tabular}

Fuente: elaboración propia

Se evidencia que ninguna de las fases del ciclo de vida de este nivel es aprobada en el nivel 2 del modelo.

\section{Evaluación de la madurez en gestión de proyectos de RSAP}

Para el nivel 1 - leguaje común no se alcanzó la calificación mínima requerida para considerar que se cumple. Con respecto al nivel 2 - procesos comunes tampoco se alcanzó el puntaje mínimo para considerar que se cumple. En este sentido, se puede establecer que RSAP no supera los niveles 1. Lenguaje común y 2. Procesos comunes del modelo. Por lo tanto, teniendo en cuenta el solapamiento de niveles planteado por Kerzner al no estar los niveles 1 y 2 aprobados en la organización, se confirma que no se han desarrollado los niveles siguientes. Por ende, RSAP se ubica en el nivel 1 "Lenguaje común” como el nivel de madurez actual en la gestión de sus proyectos. 


\section{Desarrollo modelo para la gestión de proyectos RSAP}

Dinámica en la gestión de proyectos en RSAP

El sistema actual de la empresa garantiza las condiciones mínimas del coste en los presupuestos y plazos de los proyectos, los cuales se condicionan principalmente por el cliente. Como herramientas de gestión se utilizan instrumentos ofimáticas, desde donde se gestionan ordenes de recurso humano, inventario, materias primas, logística, transporte y mano de obra, requeridas para la realización de los proyectos. El control de los proyectos está centralizado en la producción general de la organización. En las etapas de ejecución (producción) son los directores de proyectos los que se encargan de llevar el seguimiento y control a la ejecución.

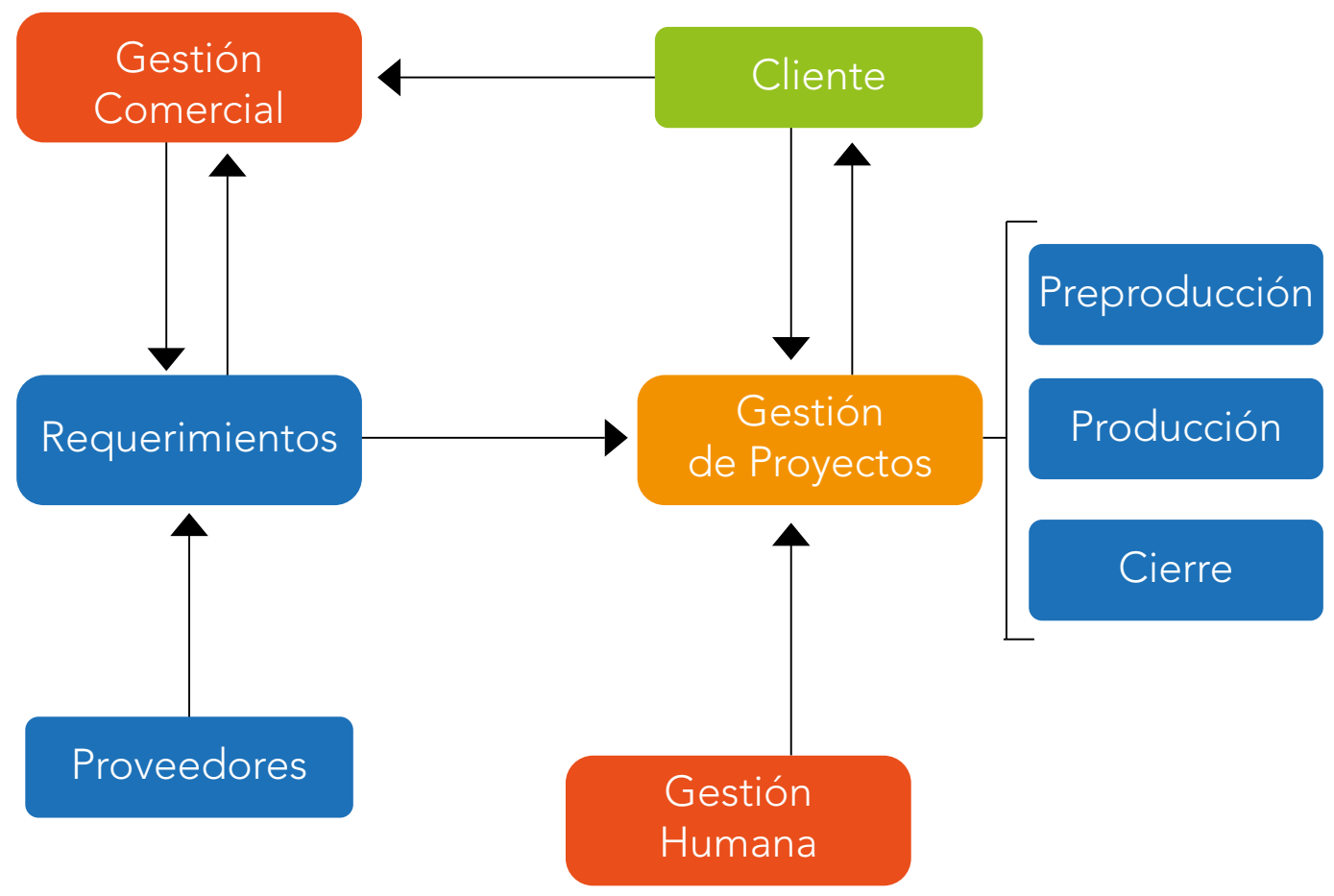

Figura 5: Actual gestión de proyectos RSAP

Fuente: elaboración propia 
En el flujo que realiza RSAP para la gestión de sus proyectos, inicialmente el cliente se acerca a solicitar el servicio. La comunicación se realiza con el área comercial de la compañía, en donde se determinan y ajustan los requerimientos específicos del servicio y se establecen los objetivos del cliente, con base en los cuales se realiza una propuesta de producción (ejecución) que incluye el coste del servicio. Internamente se toman los requerimientos y con ellos se determina la planificación del proyecto siguiendo las fases de desarrollo de proyectos comúnmente utilizada en este sector (preproducción, producción y cierre). El departamento de gestión humana alista el equipo del proyecto y se procede a realizar la ejecución del mismo. El flujo de procesos que se realiza en la actualidad en RSAP se relaciona a continuación (figura 6).

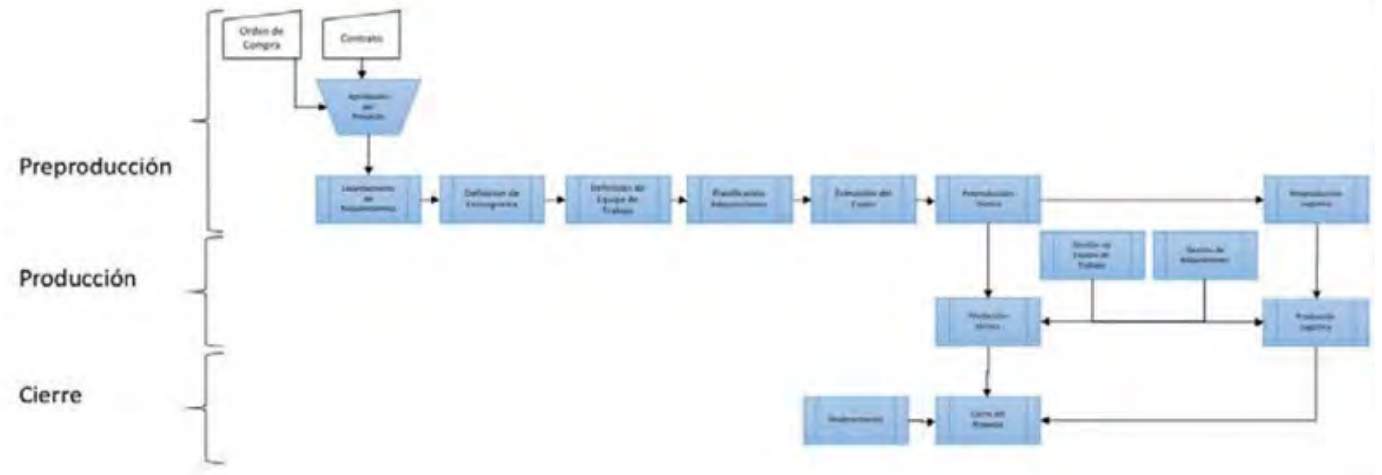

Figura 6. Flujo de procesos para la gestión de proyectos en RSAP

Fuente: RSAP, (2016)

RSAP inicia un proyecto con su etapa de preproducción, una vez el área comercial reporta una orden de compra, de servicio o un contrato. Posteriormente se procede a brindar el aval del proyecto mediante una orden de aprobación proveniente del CEO de la compañía. Luego, en continua comunicación con el cliente se realiza el levantamiento de requerimientos. Una vez son establecidos los requerimientos definitivos se procede a realizar una definición de cronograma con estimaciones de tiempo y recursos para las fases de preproducción y producción. En la fase de producción se lleva a cabo el desarrollo del proyecto. Una vez se cuente con el cronograma del proyecto se procederá a definir el equipo de trabajo que hará parte del mismo. Posteriormente se planifican las 
adquisiciones, si el proyecto las requiere, y se procede a estimar el coste del proyecto. Finalmente, en la etapa de preproducción se realizan reuniones de preproducción técnica y preproducción logística en las que se analiza la ejecución (producción) del proyecto.

Anterior a la producción se lleva a cabo la gestión del equipo de trabajo y la gestión de las adquisiciones definidas en la preproducción; con estas dos entradas se procede a realizar la producción técnica y logística del proyecto. Una vez está se ha realizado a satisfacción del cliente, se procede a dar cierre al mismo y para clientes corporativos importantes para lo organización se procede a realizar una reunión de realimentación donde se establecen directrices para proyectos futuros.

\section{Caracterización de los proyectos de RSAP}

Los proyectos de RSAP son principalmente de alquiler de sistemas de refuerzo sonoro y sistemas de iluminación profesional para eventos y conciertos, estos sistemas se alquilan a compañías de renta de audio e iluminación. La organización cuenta con sistemas de refuerzo sonoro convencionales y de tipo Line Array y sistemas de iluminación profesional. Lo anterior, permite a RSAP incluir en su portafolio de servicios la oferta de varios tipos de configuraciones e instalaciones. Por esta razón, RSAP categoriza sus proyectos en función de sus requerimientos como proyectos de pequeña, mediana y gran envergadura. Esto tiene incidencia directa en los tiempos de preproducción (planificación) y de producción (ejecución).

En la tabla 4 se referencia la categorización de cada proyecto según su envergadura y sus tiempos de planificación y ejecución promedio que en la actualidad se desarrollan en RSAP. 
Tabla 4. Categorización de proyectos en RSAP, según su envergadura

\begin{tabular}{|c|c|c|c|}
\hline Categorización & $\begin{array}{l}\text { Criterios de } \\
\text { categorización }\end{array}$ & $\begin{array}{l}\text { Tiempo } \\
\text { promedio } \\
\text { planificación }\end{array}$ & $\begin{array}{l}\text { Tiempo promedio } \\
\text { de ejecución }\end{array}$ \\
\hline $\begin{array}{l}\text { Proyectos } \\
\text { De gran } \\
\text { envergadura }\end{array}$ & $\begin{array}{l}\text { Proyectos con } \\
\text { requerimientos de más } \\
\text { de } 15.000 \text { Watts de } \\
\text { potencia sonora } \\
\text { Proyectos corporativos } \\
\text { que requieren de } \\
\text { una calidad sonora } \\
\text { superior }\end{array}$ & $\begin{array}{l}\text { Entre } 5 \text { y } 6 \\
\text { meses }\end{array}$ & $\begin{array}{l}\text { De } 1 \text { a } 3 \text { días o más, } \\
\text { según requerimientos } \\
\text { del cliente }\end{array}$ \\
\hline $\begin{array}{l}\text { Proyectos } \\
\text { de mediana } \\
\text { envergadura }\end{array}$ & $\begin{array}{l}\text { Proyectos de entre } \\
5000 \text { y } 15.000 \text { Watts de } \\
\text { potencia }\end{array}$ & $\begin{array}{l}\text { Entre } 1 \text { y } 2 \\
\text { meses }\end{array}$ & $\begin{array}{l}\text { De } 1 \text { a } 3 \text { días, o según } \\
\text { requerimientos del } \\
\text { cliente }\end{array}$ \\
\hline $\begin{array}{l}\text { Proyectos } \\
\text { de pequeña } \\
\text { envergadura }\end{array}$ & $\begin{array}{l}\text { Proyectos de menos } \\
\text { de } 5000 \text { Watts de } \\
\text { potencia }\end{array}$ & No se planifican & $\begin{array}{l}\text { Generalmente duran } \\
\text { entre } 1 \text { y } 2 \text { días, o según } \\
\text { requerimientos del } \\
\text { cliente }\end{array}$ \\
\hline
\end{tabular}

Fuente: RSAP, (2016)

Los proyectos de gran envergadura en RSAP son definidos principalmente por la cantidad de vatios (Watts) a amplificar, esto tiene una relación directa en la cantidad y volumen de equipamiento a utilizar y sus requerimiento técnicos y logísticos, lo que significa también la utilización de una mayor cantidad de recursos en general.

Por otra parte, dentro de su gestión organizacional, la empresa no realiza control ni seguimiento a los dominios económico y temporal en los proyectos. Se solicitó información de algunos proyectos desarrollados por RSAP para obtener variaciones de costo y tiempo, respecto a los plazos y presupuestos aplicables, en las etapas de preproducción. En la tabla 5 podemos encontrar la información de algunos proyectos realizados por RSAP. 
Tabla 5. Variacion de tiempo y costo de últimos proyectos de RSAP

\begin{tabular}{|c|c|c|c|c|}
\hline Proyecto & $\begin{array}{l}\text { Tiempo } \\
\text { estimado }\end{array}$ & $\begin{array}{l}\text { Costo estimado } \\
\text { (COP) }\end{array}$ & $\begin{array}{l}\text { Tiempo } \\
\text { real }\end{array}$ & $\begin{array}{l}\text { Costo real } \\
\text { (COP) }\end{array}$ \\
\hline $\begin{array}{l}\text { Rueda de prensa Yuri } \\
\text { Alveolar - Comité Olímpico } \\
\text { Colombiano }\end{array}$ & 15 días & $\$ 6.500 .000$ & 15 días & $\$ 6.700 .000$ \\
\hline $\begin{array}{l}\text { Rueda de prensa Oscar } \\
\text { Figueroa - Comité Olímpico } \\
\text { Colombiano }\end{array}$ & 15 días & $\$ 5.500 .000$ & 15 días & $\$ 6.550 .000$ \\
\hline Despedida Rio 2016 - Claro & 8 días & $\$ 4.200 .000$ & 8 días & $\$ 4.350 .000$ \\
\hline $\begin{array}{l}\text { Rueda de Prensa FIFA } 2016 \\
\text { - Federación Colombiana } \\
\text { de Futbol }\end{array}$ & 30 días & $\$ 5.500 .000$ & 30 días & $\$ 5.850 .000$ \\
\hline Cena del deporte - Coldeportes & 30 días & $\$ 6.500 .000$ & 30 días & $\$ 6.700 .000$ \\
\hline
\end{tabular}

Fuente: RSAP (s.f.).

Se evidencia que existen desviaciones en costo. Esto ocurre generalmente por falencias en la preproducción y producción de los proyectos que afectan la gestión de los mismos. Lo anterior se consolida como un factor crítico de incidencia en la ejecución de proyectos de RSAP.

Las variaciones de tiempo no se dan en este tipo de proyectos dado que la ejecución del proyecto se realiza durante fechas específicas que generalmente son establecidas por el cliente. Por lo tanto, desde el inicio del proyecto se establece un periodo para planificación y ejecución y este se debe cumplir estrictamente por parte de RSAP. Cabe resaltar que lo anterior no justifica que las acciones de trabajo en dichos proyectos hayan sido acertadas, pues no se evitó la generación de las desviaciones identificadas. A continuación se relacionan las causalidades identificadas que generan principalmente las variaciones de costo en los proyectos de RSAP en la organización.

Capacidad Funcional. Cuando varios proyectos se ejecutan de manera simultánea la capacidad funcional de RSAP se ve comprometida, pues dependiendo de la envergadura de los proyectos simultáneos, la organización puede 
llegar a copar su capacidad funcional, esto debido al equipamiento, pues el equipamiento es la principal restricción en la implementación de este tipo de proyectos.

Requerimientos funcionales. Los requerimientos están variando en la ejecución de acuerdo a las circunstancias propias del entorno o a los cambios que el cliente realiza sobre la etapa de ejecución, por lo tanto, dichos cambios ocasionan variaciones en los costos del proyecto.

Requerimientos no funcionales. Existen requerimientos no funcionales adscritos a los proyectos, los cuales muchas veces dependen de factores del entorno que afectan la planificación o ejecución de los mismos.

Comunicaciones. Una mala gestión de las comunicaciones en los proyectos impacta negativamente la gestión del alcance y ocasiona errores repetitivos en la ejecución de los mismos en RSAP.

Gestión del recurso humano. Proyectos en los que el equipo asignado para la ejecución no es suficiente o no se cuenta con el personal especializado requerido.

Gestión del riesgo. Continuamente se están materializando los riesgos en los proyectos de RSAP, lo que impide el cumplimiento del alcance o el coste definido en la etapa de preproducción o también afecta la calidad de los entregables.

Gestión del conocimiento. No se realiza una adecuada gestión del conocimiento al interior de la organización, por lo tanto no existen manuales de lecciones aprendidas ni referentes que le permita a la organización retomar experiencias anteriores en proyectos de características similares. 


\section{Roles que intervienen en los proyectos en RSAP}

En la realización de un proyecto en RSAP se identificaron los roles que hacen parte activa en su desarrollo. Estos roles son los encargados del desarrollo de los procesos que se llevan a cabo en la correcta consecución del ciclo de vida de un proyecto en RSAP. En la figura 7 se presentan los roles que intervienen en un proyecto en RSAP.

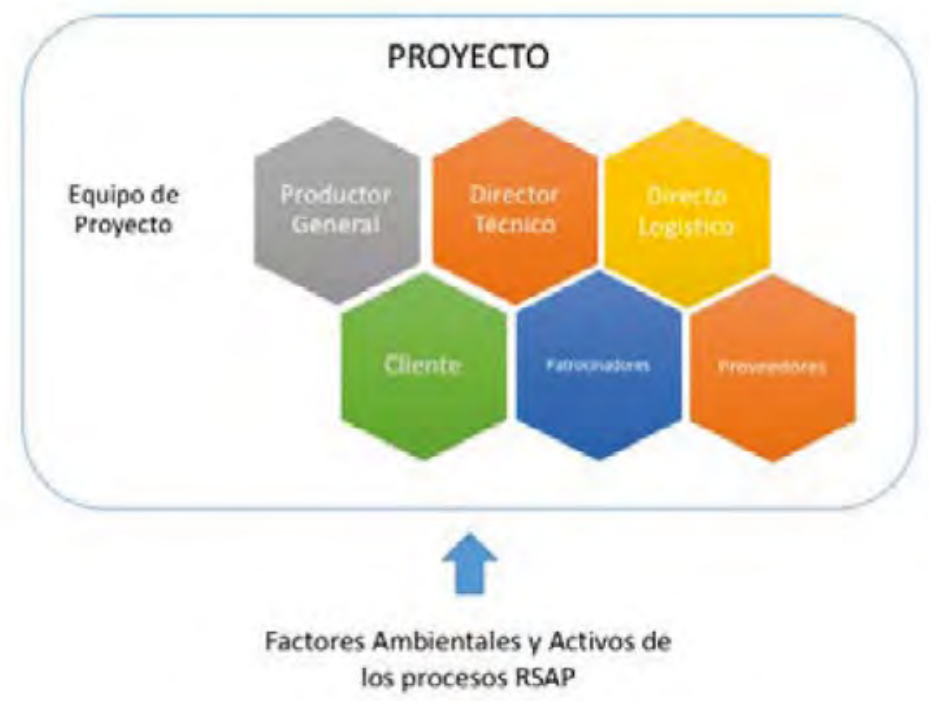

Figura 7. Roles que intervienen en un proyecto en RSAP

Fuente: elaboración propia

Equipo de proyecto. El equipo de proyecto incluye al director y al grupo de individuos que actúan conjuntamente en la realización del trabajo y en la consecución de sus objetivos (PMBOK, 2013). En RSAP, el equipo de proyecto se estructura en 3 roles: el productor general, quien es el equivalente al director de proyectos y dos roles de apoyo como lo son el director técnico y el director logístico, estos dos últimos serán los encargados de la gestión en sus enfoques respectivos. 
Productor general. Así se le denomina comúnmente en el medio del espectáculo y el entretenimiento. El productor general es la persona encargada de dirigir el trabajo en la realización de un proyecto de esta índole. Desde un enfoque de dirección de proyectos, el productor general será el equivalente al director del proyecto y dirigirá el equipo de proyecto asumiendo sus funciones y responsabilidades.

Director Técnico. Este rol se encarga de la gestión técnica en todos los ciclos de vida del proyecto. El director técnico es un experto en el enfoque técnico que el proyecto requiere, para RSAP debe ser un experto en sistemas de refuerzo sonoro e iluminación profesional.

Director logístico. Es la persona encargada de la gestión logística en el aprovisionamiento, distribución, transporte, instalación, adecuación y confort que el proyecto o las personas involucradas en él requieren. Para RSAP el director logístico deberá ser una persona experimentada en el desarrollo de proyectos de este tipo con gran conocimiento en la gestión de los recursos de RSAP.

Clientes. Son varios los clientes que en la realización de un proyecto pueden llegar a estar involucrados, estos inciden continuamente en la planificación y ejecución de los eventos, impactando el ciclo de vida de los proyectos de RSAP. Los clientes se consideran como un interesado con alto nivel de autoridad sobre los proyectos.

Proveedores. Uno o más proveedores pueden hacer parte de un proyecto en RSAP. Aunque los proveedores se consideran un interesado de bajo nivel de autoridad, este rol también hace parte del ciclo de vida de los proyectos de RSAP. 


\section{Modelo para la gestión de proyectos RSAP}

Inicialmente se define el ciclo de vida del proyecto ajustando dicho ciclo al referenciado en el PMBOK, teniendo en cuenta la denominación de etapas desarrolladas en la gestión actual de los proyectos en RSAP e incluyendo la etapa de inicio, seguimiento y control, que no son desarrolladas en la actualidad. Las fases de ciclo de vida definidas para los proyectos en RSAP se presentan en la figura 7.

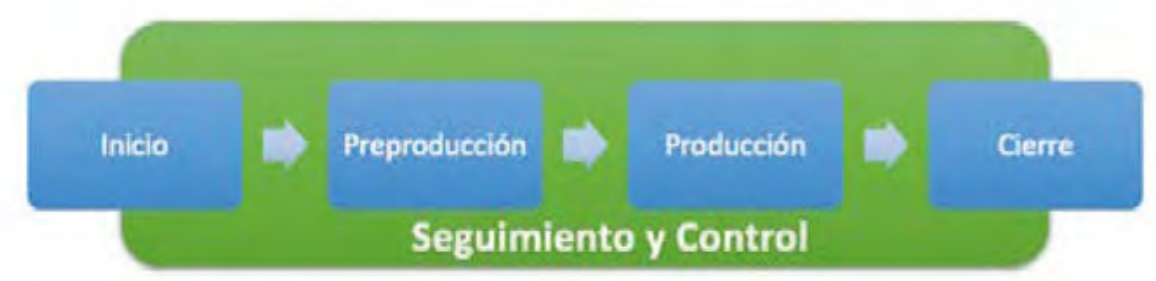

Figura 7. Fases de ciclo de vida definidas para un proyecto en RSAP Fuente: elaboración propia

Una vez se definieron las etapas del ciclo de vida del proyecto se procedió a definir los servicios específicos. Retomando la envergadura de los proyectos definidos en el tabla 4, se tomarán como proyectos en RSAP los servicios que incluyan sistemas de refuerzos sonoros de mediana y gran envergadura. Es decir, proyectos que requieran más de 5.000 Vatios (Watts) de potencia y proyectos para clientes corporativos que requieran de una alta calidad sonora. Los proyectos de pequeña envergadura, al no tener mayores requerimientos técnicos, logísticos, tiempos de preproducción y producción establecidos, no serán incluidos como proyectos aplicables al modelo.

Posteriormente, teniendo en cuenta los grupos de procesos, las áreas de conocimiento y las fases del ciclo de vida de un proyecto establecidos por el PMBOK, se procedió a realizar un análisis del flujo de procesos actual de RSAP (figura 8) y a complementar el mismo con los procesos o grupos de procesos en la áreas de conocimiento que el PMBOK establece y que serán necesarias en la gestión 
de proyectos en RSAP, en aras de garantizar el éxito del mismo en términos de la triple restricción (alcance, tiempo y costo). Producto del análisis y ajuste se obtuvo el siguiente flujo de procesos referenciado en la figura 8.

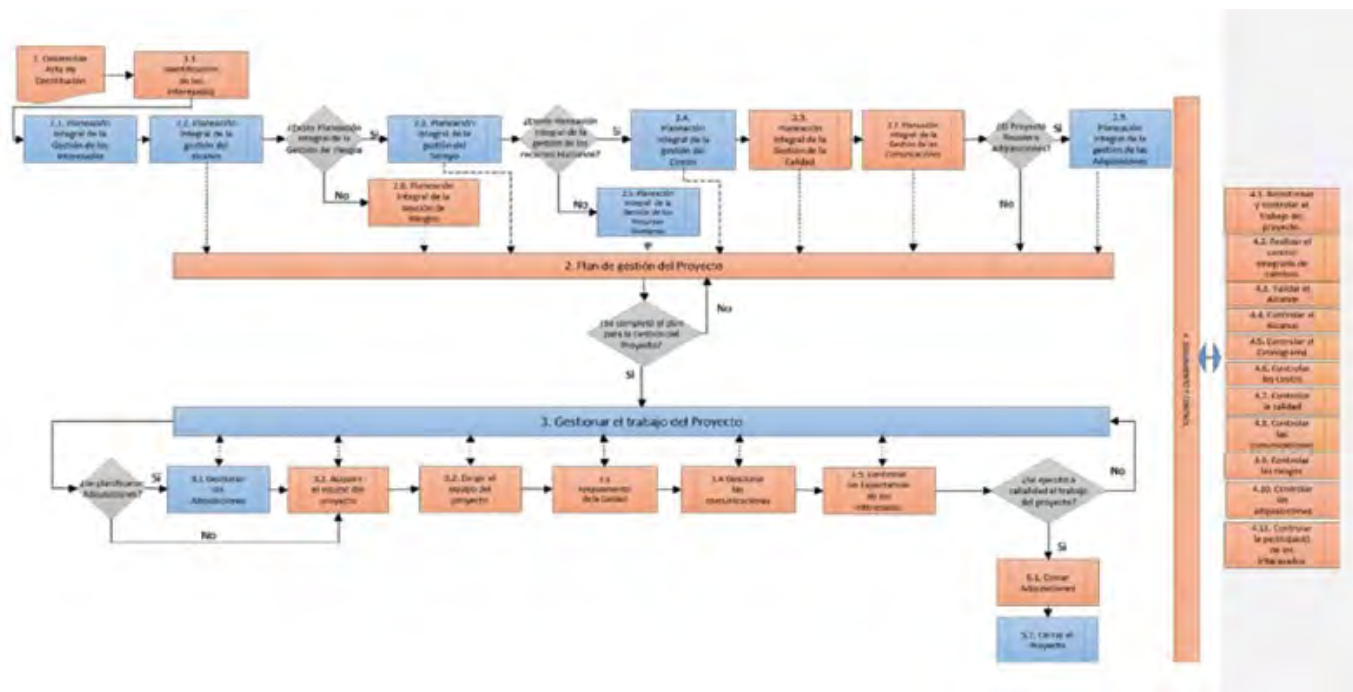

Figura 8. Modelo de gestión obtenido para los proyectos de RSAP Fuente: elaboración propia

El flujo de procesos obtenido representa los procesos o grupos de procesos que, según la guía de los fundamentos del PMI, se deben desarrollar con el fin de garantizar la correcta realización de sus proyectos. En color naranja se presentan procesos o grupos de procesos que no se realizaban en RSAP y que se deben realizar según el PMBOK, en color azul se presentan los procesos o grupos de procesos que de una u otra manera hacen parte actual de la gestión de proyectos en RSAP y en color gris se presentan los condicionales que garantizan cumplir las restricciones en la consecución lógica del desarrollo de cada fase de ciclo de vida de los proyectos.

En total se definen treinta grupos de procesos para el desarrollo del modelo, dos corresponden a la fase de inicio, once a la fase de preproducción, siete a la fase de producción, dos a la fase de cierre y once a la fase de seguimiento y control. Cabe resaltar que en cada grupo de procesos se abordan procesos que 
se proponen en el PMBOK, pero cada grupo de proceso es agrupado estratégicamente para facilitar el entendimiento del modelo en RSAP.

Posteriormente, se procedió a definir, mediante una matriz de asignación de responsabilidades RACI, el nivel de responsabilidad de cada rol que interviene en un proyecto para cada fase de ciclo de vida de proyecto. Así se garantizará la calidad de los entregables en el desarrollo de cada proceso en los proyectos en RSAP. En la tabla 6 se muestra un ejemplo de la matriz RACl para los procesos en la fase de inicio.

Tabla 6. Ejemplo de aplicación matriz RACl - Procesos de inicio

\begin{tabular}{|c|c|c|c|c|c|c|c|c|}
\hline \multicolumn{9}{|c|}{ Matriz RACI - Procesos de inicio } \\
\hline 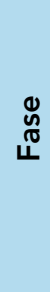 & $\begin{array}{l}\text { Grupo de } \\
\text { procesos }\end{array}$ & Proceso & 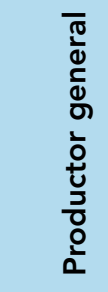 & 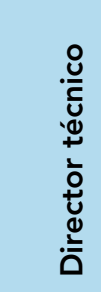 & 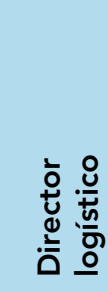 & 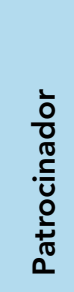 & 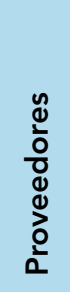 & $\underset{\frac{\Phi}{\tilde{\sigma}}}{\frac{\Phi}{U}}$ \\
\hline \multirow{2}{*}{$\stackrel{\stackrel{0}{\underline{\underline{S}}}}{\underline{\underline{\underline{S}}}}$} & $\begin{array}{l}\text { Desarrollar acta } \\
\text { de constitución }\end{array}$ & $\begin{array}{l}\text { Desarrollar acta } \\
\text { de constitución }\end{array}$ & $R$ & C & C & A & 1 & I \\
\hline & $\begin{array}{l}\text { Identificar a los } \\
\text { interesados }\end{array}$ & $\begin{array}{l}\text { Realizar la iden- } \\
\text { tificación de los } \\
\text { interesados }\end{array}$ & A & $\mathrm{R}$ & $\mathrm{R}$ & C & 1 & 1 \\
\hline
\end{tabular}

Fuente: elaboración propia

Por ejemplo, el responsable del proceso "desarrollar acta de constitución" será el productor general; así mismo, los directores técnicos y logísticos serán consultados; mientras que el patrocinador del proyecto será la persona responsable de velar por que la actividad se realice, y finalmente los proveedores y clientes serán informados de la realización y el resultado del proceso. 
Adicionalmente, también se presenta para cada fase de ciclo de vida de proyecto una descripción detallada de cada proceso propuesto, los insumos requeridos (entradas) y los productos a obtener (salidas). En la tabla 7 se presenta un ejemplo de lo anteriormente expuesto.

Tabla 7. Descripción de procesos fase de inicio - Proyectos RSAP

\begin{tabular}{|c|c|c|c|c|c|}
\hline 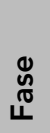 & $\begin{array}{l}\text { Grupo de } \\
\text { procesos }\end{array}$ & $\begin{array}{l}\stackrel{\circ}{\mathscr{y}} \\
\stackrel{0}{0} \\
\stackrel{0}{0}\end{array}$ & Descripción & $\begin{array}{l}\text { Insumos } \\
\text { requeridos }\end{array}$ & $\begin{array}{l}\text { Productos } \\
\text { a obtener }\end{array}$ \\
\hline & $\begin{array}{l}\text { Desarrollar } \\
\text { acta de } \\
\text { constitución }\end{array}$ & 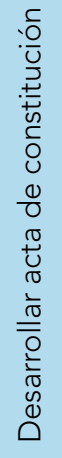 & $\begin{array}{l}\text { Mediante la elaboración } \\
\text { de un documento se debe } \\
\text { reconocer formalmente la } \\
\text { existencia de un proyecto } \\
\text { y conferir al director de } \\
\text { proyectos la autoridad } \\
\text { para asignar los recursos } \\
\text { RSAP a las actividades del } \\
\text { proyecto }\end{array}$ & $\begin{array}{l}\text { Factores } \\
\text { ambientales de la } \\
\text { empresa } \\
\text { Activos de la } \\
\text { organización } \\
\text { Orden de compra } \\
\text { Orden de servicio } \\
\text { Contrato } \\
\text { Aprobación del } \\
\text { patrocinador. }\end{array}$ & $\begin{array}{l}\text { Acta de } \\
\text { constitución } \\
\text { del proyecto }\end{array}$ \\
\hline$\frac{\text { 음 }}{\underline{\underline{v}}}$ & $\begin{array}{l}\text { Identificar } \\
\text { a los } \\
\text { interesados }\end{array}$ & 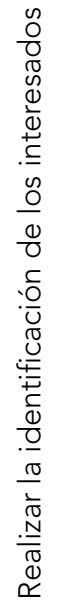 & $\begin{array}{l}\text { Se debe llevar a cabo } \\
\text { la identificación de } \\
\text { los actores (personas } \\
\text { y organizaciones) que } \\
\text { pueden influir o impactar } \\
\text { en la toma de decisiones } \\
\text { en el proyecto, sus } \\
\text { entregables o el desarrollo } \\
\text { y éxito del mismo }\end{array}$ & $\begin{array}{l}\text { Acta de constitución } \\
\text { del proyecto } \\
\text { Documentos de las } \\
\text { adquisiciones } \\
\text { Factores } \\
\text { ambientales de la } \\
\text { empresa } \\
\text { Activos de los } \\
\text { procesos de la } \\
\text { organización }\end{array}$ & $\begin{array}{l}\text { Registro de } \\
\text { interesados }\end{array}$ \\
\hline
\end{tabular}

Fuente: elaboración propia 


\section{Validación del modelo}

Tomando como referente un estudio denominado "Validez de contenido y juicio de expertos: Una aproximación a su utilización", desarrollado por Jazmine Escobar y Ángela Cuervo, el cual se centra en la validez del objetivo por medio de la utilización de juicio de expertos como parte del proceso de estimación de dicha validez (Escobar y Cuervo, 2008), se procedió a realizar la validación por expertos del modelo desarrollado. De esta manera, aplicando la metodología referenciada por el estudio, se diseñó y validó un instrumento para aplicarlo a ocho expertos. Los expertos que participaron en el proceso de validación se evidencian en la tabla 8.

Tabla 8. Perfil y experiencia de expertos seleccionados

\begin{tabular}{|c|c|c|c|}
\hline & Perfil & Experiencia & Áreas de experiencia profesional \\
\hline $\begin{array}{c}\text { Experto } \\
1\end{array}$ & $\begin{array}{l}\text { Ingeniero electrónico, } \\
\text { magíster en } \\
\text { teleinformática }\end{array}$ & 20 años & $\begin{array}{l}\text { Gerencia de proyectos, TIC, seguridad de la } \\
\text { información y continuidad de negocio }\end{array}$ \\
\hline $\begin{array}{l}\text { Experto } \\
\quad 2\end{array}$ & $\begin{array}{l}\text { Ingeniero electrónico, } \\
\text { MBA }\end{array}$ & 32 años & Estrategia, gerencia, telecomunicaciones \\
\hline $\begin{array}{l}\text { Experto } \\
3\end{array}$ & $\begin{array}{l}\text { Ingeniero de } \\
\text { sistemas, especialista } \\
\text { en gerencia de } \\
\text { proyectos, MBA }\end{array}$ & 35 Años & $\begin{array}{l}\text { Gerencia de proyectos, sistemas } \\
\text { de gestión de la calidad, auditoria } \\
\text { informática, estructuras organizacionales e } \\
\text { implementación de PMO }\end{array}$ \\
\hline $\begin{array}{l}\text { Experto } \\
4\end{array}$ & $\begin{array}{l}\text { Ingeniero de } \\
\text { sistemas, maestría en } \\
\text { sistemas } \\
\text { de información }\end{array}$ & 14 años & $\begin{array}{l}\text { Planeación estratégica de TI, Gobierno de } \\
\text { TI, arquitectura empresarial, gerencia de } \\
\text { proyectos }\end{array}$ \\
\hline $\begin{array}{l}\text { Experto } \\
\quad 5\end{array}$ & $\begin{array}{l}\text { Producción técnica } \\
\text { de eventos }\end{array}$ & 10 años & Producción técnica de eventos, BTL \\
\hline $\begin{array}{l}\text { Experto } \\
6\end{array}$ & $\begin{array}{l}\text { Licenciado en } \\
\text { electrónica }\end{array}$ & 15 años & $\begin{array}{l}\text { Sonido en vivo, diseño de sistemas } \\
\text { electroacústicos, sistemas de refuerzo sonoro }\end{array}$ \\
\hline $\begin{array}{l}\text { Experto } \\
7\end{array}$ & $\begin{array}{l}\text { Ingeniero de sonido, } \\
\text { especialista en } \\
\text { producción musical }\end{array}$ & 13 años & $\begin{array}{l}\text { Producción técnica de eventos, producción } \\
\text { musical, sistemas de refuerzo sonoro, sonido } \\
\text { en vivo }\end{array}$ \\
\hline $\begin{array}{l}\text { Experto } \\
8\end{array}$ & $\begin{array}{l}\text { Músico, empresario, } \\
\text { abogado }\end{array}$ & 27 años & $\begin{array}{l}\text { Producción técnica de eventos, desarrollo } \\
\text { organizacional, sistemas de refuerzo sonoro, } \\
\text { gestión de eventos y conciertos }\end{array}$ \\
\hline
\end{tabular}

Fuente: elaboración propia 
Según lo que establece el estudio en mención, se definieron las categorías sobre las cuales se evaluó el modelo desarrollado (tabla 9).

Tabla 9. Criterios definidos para categorías - Instrumento de validación

\begin{tabular}{ll}
\hline Categoría & \multicolumn{1}{c}{ Criterio } \\
\hline Suficiencia & $\begin{array}{l}\text { El modelo dispone de información suficiente para que la } \\
\text { organización bajo estudio pueda implementarlo en un } \\
\text { proyecto }\end{array}$ \\
\hline Claridad & $\begin{array}{l}\text { El modelo posee la claridad suficiente para que la } \\
\text { organización bajo estudio pueda implementarlo }\end{array}$ \\
\hline Coherencia & $\begin{array}{l}\text { El modelo es coherente con este tipo de proyectos y con } \\
\text { las buenas prácticas del PMI }\end{array}$ \\
\hline Relevancia & $\begin{array}{l}\text { El modelo es relevante con este tipo de proyectos y con } \\
\text { las buenas prácticas del PMl }\end{array}$ \\
\hline
\end{tabular}

Fuente: elaboración propia

\section{Resultados y validación del modelo}

A continuación se presentan los resultados, con su respectivo análisis, obtenidos de la validación del modelo desarrollado. En las figuras 9 y 10 se presentan los resultados para las categorías de suficiencia y claridad.

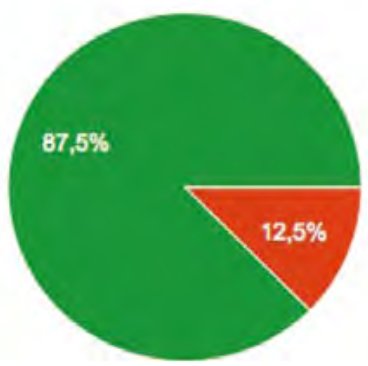

No cumple con el criterio

Bajo Nivel de cumplimiento

Moderado Nivel de Cumplimiento

Cumple con el criterio

Figura 9. Categoría suficiencia

Fuente: elaboración propia 
En la categoría de suficiencia para el criterio "El modelo dispone de información suficiente para que la organización bajo estudio pueda implementarlo en un proyecto" el $87,5 \%$ de los expertos consultados consideraron que este se cumple. Por otra parte, el $12,5 \%$ de los expertos consultados consideran que este ítem tiene un bajo nivel de cumplimiento. En este sentido se evidencia que el modelo desarrollado contiene la información suficiente para cumplir con su objetivo.
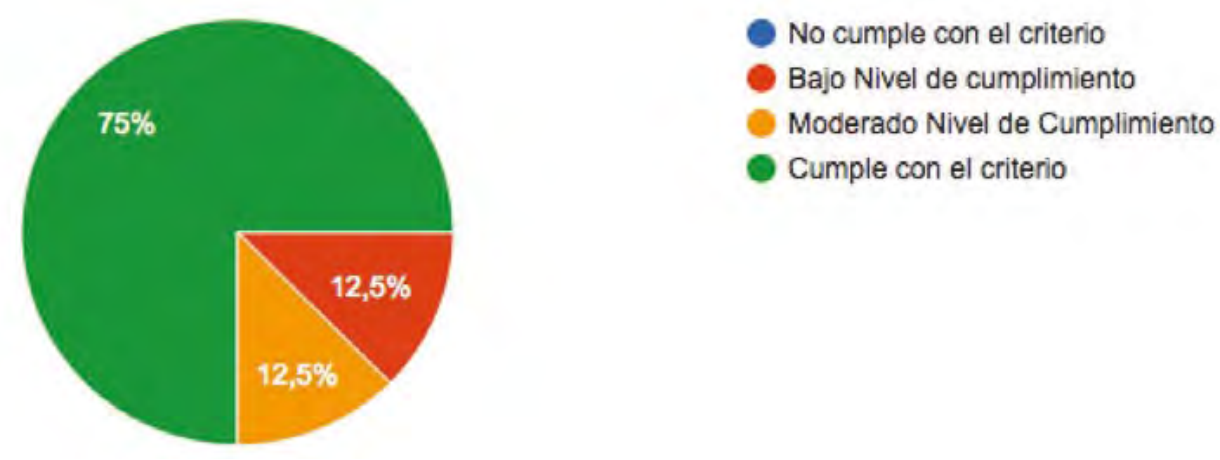

Figura 10. Categoría claridad

Fuente: elaboración propia

Para la categoría de claridad, en el criterio "El modelo posee la claridad suficiente para que la organización bajo estudio pueda implementarlo", un 75\% de los expertos considera que este se cumple. Adicionalmente, un 12,5\% de los expertos consultados considera que el modelo requiere alguna modificación específica de contenido y el $12,5 \%$ restante considera que el modelo no posee la claridad requerida para cumplir con el objetivo. Si bien no se alcanzó el $80 \%$ requerido para considerar la aprobación de esta categoría, se evidencia que una gran proporción de los expertos consultados (75\%) están de acuerdo con que el criterio de esta categoría se cumple y una $12,5 \%$ que existe un moderado nivel de cumplimiento. Por lo tanto, se tienen en cuenta las observaciones específicas de los expertos que no están de acuerdo con este criterio para tomar acciones de mejora sobre el modelo desarrollado y así atender dichas observaciones, con el fin de asegurar el grado de cumplimento requerido. 


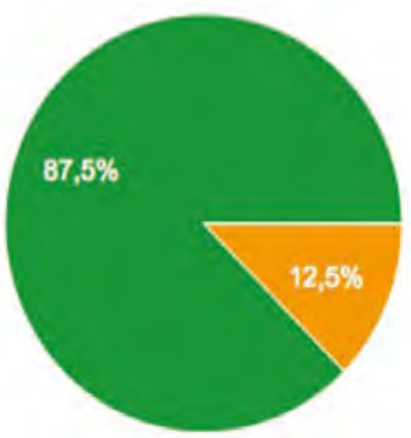

Figura 11. Categoría coherencia
No cumple con el criterio

Bajo Nivel de cumplimiento

Moderado Nivel de Cumplimiento

Cumple con el criterio

Fuente: elaboración propia

En la categoría de coherencia para el criterio "El modelo es coherente con este tipo de proyectos y con las buenas prácticas del PMI", el 87,5\% de los expertos considera que se cumple, y el $12 \%$ que se cumple con un moderado nivel de cumplimiento. Se evidencia un cumplimiento superior al $80 \%$, lo cual da cuenta de que el modelo se encuentra completamente relacionado con el objetivo del mismo.

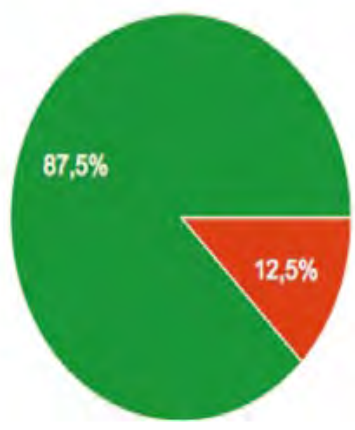

No cumple con el criterio

Bajo Nivel de cumplimiento

Moderado Nivel de Cumplimiento

Cumple con el criterio

Figura 12. Categoría relevancia

Fuente: elaboración propia

Por otra parte, en la categoría de relevancia para el criterio"El modelo es coherente con este tipo de proyectos y con las buenas prácticas del PMI", el 87,5\% de los expertos consultados considera que este se cumple, mientras que el $12,5 \%$ restante considera que este criterio tiene un bajo nivel de cumplimiento. En este sentido, se puede afirmar que el modelo es relevante y puede ser usado por la pyme estudiada. 


\section{Discusión}

Según los resultados obtenidos en la validación de expertos, se evidencia que el modelo desarrollado responde a la implementación de las buenas prácticas que establece el PMI; adicionalmente, contiene la información y claridad suficiente para lograr ser implementado en los proyectos de la pyme RSAP. Esto último, dado que el alcance propuesto para el desarrollo de cada grupo de procesos del modelo tiene en cuenta el perfil de madurez actual de la pyme, sus conocimientos y herramientas disponibles. En este sentido, con los recursos actuales de la pyme se podría pasar el modelo desarrollado a una etapa de implementación. Lo anterior permitiría la correcta apropiación -por parte de la pyme- de los conocimientos establecidos como buenas prácticas por el PMI, orientando así su desarrollo de madurez en gestión de proyectos y la mejora en sus procesos.

El modelo desarrollado propone la implementación de dos fases de ciclo de vida de proyecto (inicio, seguimiento y control) inexistentes en la actualidad para la gestión de los proyectos de RSAP, y fortalece, a nivel de procesos, las fases ya establecidas en la actualidad (preproducción, producción y cierre), logrando, de esta manera, abordar la totalidad de áreas del conocimiento y sus respectivos procesos establecidas por el PMI.

Para los procesos o grupos de procesos que en la actualidad no se llevan a cabo en la PYME se propone la guía de fundamentos para la dirección de proyectos del PMI (quinta edición) como manual para ser apropiado y conceptualizado por la cultura organizacional de la PYME. Esto permitirá que la organización ingrese en el paradigma de la gestión de proyectos desde el enfoque del PMI. Ahora bien, una vez que la RSAP logre superar el nivel de madurez actual (nivel 1 - lenguaje común) se debería ajustar el modelo en función de las mejores prácticas y evaluar implementaciones tecnológicas y de infraestructura con el fin de garantizar la orientación hacia dichas mejores prácticas. Como estudio futuro, queda abierto el trabajo para la implementación y evaluación del perfil de madurez posterior a la apropiación del modelo desarrollado en la pyme RSAP. 


\section{Conclusiones}

- Los modelos de madurez para la gestión de proyectos miden las capacidades de la organización frente a un estándar, en función de las buenas prácticas en la gestión de proyectos; sin embargo es importante analizar el modelo a implementar de acuerdo con el contexto organizacional. Debido a que no todos los modelos son aplicables a las pymes, se debe tener en cuenta que una pyme posee ciertas restricciones que define la aplicabilidad de ciertos modelos de madurez en la gestión de proyectos.

- Para el desarrollo de un modelo para la gestión de proyectos en una organización en específico se hace necesario medir su perfil actual de madurez, esto conlleva definir el alcance del modelo y nivel de profundización en la cual se va a proponer. Desconocer el perfil de madurez significaría desarroIlar un modelo que no responde a las necesidades específicas de la organización. En este sentido, desarrollar un modelo para la gestión de proyectos, teniendo en cuenta el perfil de madurez, no solo se convierte en una hoja de ruta que garantiza el éxito de los proyectos sino que es un mecanismo que conlleva que las organizaciones empiecen a superar los niveles de madurez en busca de la mejora continua y la obtención de los niveles más altos de madurez en la gestión de proyectos.

- Se logra diagnosticar el perfil de madurez en la gestión de proyectos de RSAP según los instrumentos, metodología y análisis planteados por el Modelo de Harold Kerzner. La implementación de dicho modelo es totalmente aplicable a pymes del sector y el acceso a la información del modelo se encuentra abierta y disponible, consolidándolo como un modelo apto para la aplicabilidad en pymes.

- Se desarrolla un modelo de gestión de proyectos acorde con el perfil de madurez de la RSAP, sus recursos y herramientas de gestión disponibles. Este modelo permitirá a RSAP ingresar al paradigma de las buenas prácticas de la gestión de proyectos desde el enfoque del PMI. 
- La pyme estudiada solo implementaba el $27 \%$ de los procesos que sugiere el PMI para el desarrollo de un proyecto. El modelo desarrollado incorpora los 5 grupos de procesos, 46 de los 47 procesos y las 10 áreas del conocimiento sugeridas por el PMI para la correcta gestión de un proyecto.

\section{Referencias}

Buchtik, L. (2012). Secretos para dominar la gestión de riesgos en proyectos.

Montevideo, Uruguay: Buchtik Global.

Crowe, A. (2011). The PMP Exam. Velociteach. Specialization and the Project Manager. [en línea]. Disponible en: https://www.velociteach.com/2011/09/specialization-andthe-project-manager/

Etkin, J. (2009). Gestión de la complejidad en las organizaciones. Buenos Aires, Argentina: Ediciones Granica S.A.

Escobar, J. y Cuervo, A. (2008). Validez de contenido y juicio de expertos: Una aproximación a su utilización. En Avances en Medición, pp. 27-36.

Guerrero, G. (2013). Metodología para la gestión de proyectos bajo los lineamientos del Project Management Institute en una empresa del sector eléctrico. Bogotá, D.C., Colombia: Universidad Nacional de Colombia.

Jiménez, R. (1998). Metodología de la investigación. Elementos básicos para la investigación clínica. La Habana: Ciencias Médicas.

Johnson, T. (2007). PMP Exam Success Series: Certification Exam Manual. Texas: Crosswind. Project Management Inc. 564 p. 
Kerzner, H. R. (2002). Strategic Planning for Project Management Using a Project Management Maturity Model. Unites States of America: John Wiley and Sons.

Lazaro, D., Laverde, J. y Guacheta, K. (2013). Evaluación de madurez en gestión de proyectos en las organizaciones. Aplicación en empresa del sector de servicios de Bogotá. Bogotá: Universidad EAN.

Lopéz, B. (2013). Modelos de madurez en la amisnitración de proyectos [en línea]. Disponible en http://www.ucipfg.com/Repositorio/MAP/MAPD-02/UNIDADES_DE_ APRENDIZAJE/UNIDAD_4/LIBRO_4/DOCUMENTOS/Modelos_de_Madurez_en_la_ Administracion_de_Proyectos.pdf

Mintzberg, H. (1984). La estructuración de las organizaciones. España: Editorial Ariel.

Mulcahy, R. (2009). PMP Exam Prep. United States of America. RMC Publications Inc. P. 535.

Monje, C. (2011). Metodología de la investigación cuantitativa y cualitativa. Guía Didáctica. Neiva, Hulia, Colombia: Universidad Surcolombiana.

Nokes, S. (2006). La guía definitiva de la gestión de proyectos (FT/PH). Londres: Financial Times. Prentice Hall.

PMBOK. (2013). Fundamentos para la dirección de proyectos. $5^{\circ}$ edición. Newtown Square - Pensilvania: Project Management Institute, Inc.

PMI. (2003). Organizational Project Management Maturity Model - OPM3. Newtown Square, Penssylvania, USA: Project Management Institute.

PMI. (2013). Guía de los fundamentos para la dirección de proyectos. $5^{\circ}$ edición. Newtown Square, Pensilvania, EE:UU: Project Management Institute.

PMI. (2014). Project Management Institute - Latinoamerica [en línea]. Disponible en https://americalatina.pmi.org 
Rivas, L. (2002). Nuevas formas de organización. En Estudios Gerenciales, núm. 82, pp. 13-45. Universidad ICESI. Cali, Colombia.

Rodríguez, S., Aguilar, A. y Sanchez, L. (2012). Metodología para la gestión de riesgos en proyectos. Madrid, España: Universidad Autónoma de Madrid.

Sánchez, R. (15 de 04 de 2015). Entrevista a Ruffo Sánchez Puentes. Bogotá, Colombia.

Sánchez, L., y Solarte, L. (2013). Gerencia de proyectos y estrategia organizacional: el modelo de madurez en Gestión de Proyectos CP3MO V5.0. En Revista Innovar Journal. Revista de Ciencias Administrativas y Sociales.

Thierauf, R. (1985). Investigación de Operaciones. (L. Wiley, Ed.) México: (s.e.).

Villegas, D., y lván, T. (2010). Las pymes: Una mirada a partir de la experiencia académica del MBA. En Revista MBA Eafit, 86-101. 